\title{
Review of: "Y chromosomal noncoding RNAs regulate autosomal gene expression via piRNAs in mouse testis"
}

Pang Qianqian

Potential competing interests: The author(s) declared that no potential competing interests exist.

1. Page 2, "Subsequently multicopy transcripts such as $Y 353 / B$, spermiogenesis-specific transcript on the $Y$ (Ssty) and Sycp3-like, Y-linked (Sly) from mouse Yq were projected as putative candidate genes for male sterility and spermiogenesis in mice [2, 15, 17-19]." Incorrect punctuation.

2. Is the genetic background of the mouse model "XYRIII qdel" and "B10.BR-Ydel" clear? Are there other gene deletions besides the $\mathrm{Y}$ chromosome?

3. Page 7, Please mark the " $X Y^{R I I I}$ as normal mice when it first appears from misunderstanding.

4. The additional file 10: Fig. S5A, the tubulin band in western blot and northern blot are a little fuzzy, and D3 did not have the error bar. It would be better to change a clearer image for more certain.

5. Fig4, the distortion of the electrophoresis direction and the clipping of the image lead to a weaker explanation. It is recommended to provide an unprocessed original image and statistical data of repeated experiments..

6. Page 7, "Many proteins coded by autosomal genes are deregulated in XYRIIlqdel sperm proteome", this conclusion cannot be reached without cell experiment and rescue experiment verification. 